\title{
Riqueza e composição de Arctiinae (Lepidoptera, Erebidae) em cinco Unidades de Conservação do Cerrado
}

\author{
Carolina Moreno', Flávio C. Magalhães², Luiz H. G. de Rezende², \\ Karen Neves ${ }^{3} \&$ Viviane G. Ferro ${ }^{4}$
}

\begin{abstract}
1. Programa de Pós-Graduação em Ecologia e Evolução, Departamento de Ecologia, Instituto de Ciências Biológicas, Universidade Federal de Goiás, Caixa Postal 131, 74001-970 Goiânia, GO, Brasil. (s.moreno.carol@gmail.com)

2. Curso de Ciências Biológicas, Instituto de Ciências Biológicas, Universidade Federal de Goiás, Caixa Postal 131, $74001-970$ Goiânia, GO, Brasil. (hgrluiz@gmail.com; flavio.c.3@hotmail.com)

3. Programa de Pós-Graduação em Ecologia e Conservação de Recursos Naturais, Instituto de Biologia, Universidade Federal de Uberlândia, $38400-902$ Uberlândia, MG, Brasil. (neves.karen.f@gmail.com)

4. Departamento de Ecologia, Instituto de Ciências Biológicas, Universidade Federal de Goiás, Caixa Postal 131, 74001-970, Goiânia, GO, Brasil. (vivianegferro@gmail.com)
\end{abstract}

\begin{abstract}
Richness and composition of Arctiinae (Lepidoptera, Erebidae) in five Conservation Units in the Cerrado biome. The Cerrado biome contains about $50 \%$ of the Brazilian Arctiinae moths. However, northern region of the Cerrado is under-sampled for these insects, where there are more preserved areas. Here, we described the richness, composition, abundance, and beta diversity of Arctiinae in different vegetation types in five Protected Areas in the Cerrado northeast. We found 83 species, almost $78 \%$ of them occurred in one Protected Area. Less preserved localities had higher species richness. This result can be explained as a response to frequent intermediate disturbances in the landscape of these places. Forested areas were richer in species, supporting the environmental heterogeneity hypothesis. Compositional similarity was low among protected areas and among vegetation types. More complete inventories are required to assess which factors are influencing species richness and composition patterns of Arctiinae moths in these localities and thus guide future conservation actions.
\end{abstract}

KEYWORDS. Protected Areas, inventories, moths.

RESUMO. Cerca de 50\% da diversidade de mariposas Arctiinae do Brasil está no Cerrado. Contudo, a fauna desses insetos é extremamente subamostrada na região norte do bioma, onde localizam-se as áreas de vegetação mais preservadas. Diante disso, este trabalho teve como objetivos descrever a riqueza, abundância, composição e diversidade beta de Arctiinae em diferentes fitofisionomias de cinco Unidades de Conservação localizadas no nordeste do Cerrado. Foram amostradas 83 espécies, das quais quase $78 \%$ ocorreram em apenas uma Unidade de Conservação. As localidades com menor grau de preservação apresentaram maior riqueza de espécies, o que pode ser explicado como uma resposta a distúrbios intermediários na paisagem destes locais. As fitofisionomias florestais foram mais ricas em espécies, corroborando a hipótese da heterogeneidade ambiental. De maneira geral, a similaridade da fauna foi baixa tanto entre as Unidades de Conservação quanto entre as fitofisionomias. Inventários mais completos precisam ser feitos a fim de avaliarmos que fatores estão influenciando os padrões de riqueza e composição de Arctiinae nestas localidades de Cerrado e, assim, subsidiar futuras ações de conservação.

PALAVRAS-CHAVE. Áreas protegidas, inventários, mariposas.

Arctiinae é composta de aproximadamente 11 mil espécies de mariposas, das quais seis mil são encontradas na Região Neotropical (HEPPNER, 1991) e 1.391 no Brasil (FERRo \& Diniz, 2010). No Cerrado são registradas 723 espécies (FERro et al., 2010).

Segundo Ferro et al. (2010), menos de 60\% das quadrículas de $1^{\circ}$ de latitude/ longitude que cobrem o Cerrado apresentam registros de coleta de Arctiinae, sendo que apenas a quadrícula da região de Brasília foi adequadamente inventariada. A região norte do bioma, apesar de ser extremamente subamostrada (FERRO et al., 2010), abriga as áreas com vegetação mais preservada (CAVALCANTI \& Joly, 2002). Contudo, esse panorama pode ser alterado em um futuro próximo devido à expansão da fronteira agrícola para essa região (CAVALCANTI \& JOLY, 2002). Logo, é necessário que se invista mais em inventários na parte norte do Cerrado. Através do conhecimento da diversidade dessa região será possível, por exemplo, aplicar análises de modelagem de nicho para prever áreas prioritárias para conservação.

O bioma Cerrado é composto por um mosaico de fitofisionomias. De acordo com Oliveira-Filho \& RATTER (2002), as fitofisionomias que compreendem o cerrado sensu lato são o campo limpo, campo sujo, campo cerrado, cerrado sensu stricto e cerradão. Nesta sequência, estas formações vegetais representam um gradiente de vegetação que aumenta em densidade de arbóreas e diminui em densidade de herbáceas e subarbustos (OliveiraFilho \& RatTer, 2002). No bioma Cerrado também ocorrem formações florestais que não são consideradas pertencentes ao cerrado sensu lato como, por exemplo, as matas estacionais decíduas e semidecíduas, as matas sempre verdes, as matas de vale e as matas de galeria (Oliveira-Filho \& RatTer, 2002).

Os objetivos deste trabalho são: (1) descrever a riqueza, composição, abundância e diversidade beta das espécies noturnas de Arctiinae em cinco localidades (Unidades de Conservação) na região nordeste do Cerrado e (2) avaliar a similaridade da fauna de Arctiinae entre diferentes fitofisionomias do bioma. Espera-se que as fitofisionomias mais complexas apresentem uma riqueza maior de espécies. 


\section{MATERIAL E MÉTODOS}

Amostragens. As mariposas foram amostradas em cinco Unidades de Conservação (UCs) de Cerrado: Parque Estadual do Araguaia, Parque Nacional das Nascentes do Rio Parnaíba, Estação Ecológica Serra Geral do Tocantins, Refúgio de Vida Silvestre das Veredas do Oeste Baiano e Parque Nacional Grande Sertão Veredas (Tab. I). Em cada UC foram amostrados 12 sítios abrangendo diferentes fitofisionomias do bioma (Tab. I). Somente as fitofisionomias mais representativas de cada UC foram avaliadas, de modo que cada UC foi representada nas análises por pelo menos duas formações vegetais distintas.

Em cada sítio (unidade de amostragem), as mariposas foram coletadas a partir do anoitecer até o amanhecer, durante duas noites consecutivas, com armadilha luminosa do tipo Luiz de Queiroz (Silveira-Neto \& SilveIRA, 1969) equipada com lâmpada ultravioleta de $15 \mathrm{~W}$ ligada a bateria automotiva e acoplada a uma barraca de voal. Os exemplares foram sacrificados com o uso de frascos mortíferos contendo hidróxido de amônio. Os indivíduos foram identificados através da comparação de imagens digitais de espécimes da Coleção Vitor Becker (as espécies desse acervo foram identificadas por comparação com os tipos) e da literatura (HAMPSON, 1898, 1900, 1901, 1914; Watson \& GoOdger, 1986; Piñas-Rubio et al., 2000; Piñas-Rubio \& ManZano, 2003). Os espécimes estão depositados na Coleção Zoológica da Universidade Federal de Goiás (Goiânia, GO) e na Coleção Entomológica da Universidade de Brasília (Brasília, DF).

Análise dos dados. Foram utilizados os estimadores de riqueza não-paramétricos Jackknife de primeira ordem e Chao de segunda ordem para estimar a riqueza total de Arctiinae em cada UC, e em cada fitofisionomia. Para que a variação na riqueza de espécies fosse controlada em relação ao número de indivíduos coletados, a medida de riqueza foi padronizada por curvas de rarefação baseadas no número de indivíduos, em que cada ponto de coleta foi considerado uma amostra diferente.

A diferença na composição de espécies foi testada através de uma análise de escalonamento multidimensional não-métrico (NMDS), utilizando o índice de Bray-Curtis como medida de distância. A matriz de abundância das espécies foi padronizada com raiz quadrada para diminuir as discrepâncias entre as diferentes abundâncias de cada espécie. A diferença estatística na composição de espécies entre as formações vegetais e entre as Unidades de Conservação foi avaliada com a análise de similaridade (ANOSIM).

Foi avaliado também se a diferença na riqueza de mariposas Arctiinae era devido às diferenças entre as fitofisionomias, ou devido às diferenças entre as Unidades de Conservação. Para isso, foi realizado um Modelo Linear Generalizado (GLM) com a distribuição de Poisson, que é indicada para dados de contagem (BOLKER, 2007). A seleção do melhor modelo baseou-se no critério de Akaike (AIC).

Devido ao número reduzido de indivíduos, as fitofisionomias campo sujo e vereda foram excluídas das análises de rarefação, NMDS, ANOSIM e GLM. As curvas de rarefação foram conduzidas com o software PAST (HAMmER et al., 2001), e as demais análises com o programa R (The R Development Core Team, 2010).

\section{RESULTADOS}

No total, foram amostradas 616 mariposas representadas por 83 espécies (Tab. II). Chrysostola nivaca (Jones, 1994) e Heliura tetragramma (Walker, 1854) foram as espécies mais abundantes, com 210 e 67 indivíduos, respectivamente.

UCs. O Parque Nacional Grande Sertão Veredas apresentou o maior número de indivíduos, seguido do Parque Nacional das Nascentes do Rio Parnaíba (Tab. II). As faunas dos Parques Estadual das Nascentes do Rio Parnaíba, Estadual do Araguaia e Nacional Grande Sertão Veredas foram as mais ricas em espécies (Tab. III). Contudo, quando a riqueza foi padronizada pelo número de indivíduos através da rarefação, as faunas do Parque Estadual do Araguaia e do Refúgio de Vida Silvestre das Veredas do Oeste Baiano foram significativamente mais ricas em relação às demais Unidades de Conservação (Fig. 1).

Chrysostola nivaca (Jones, 1994), Idalus carinosa (Schaus, 1905) e Dycladia lucetius (Stoll, 1781) foram encontradas em todas as cinco UCs. Entretanto, 64 espécies (77.1\% do total) estiveram presentes em apenas uma UC. O Parque Estadual do Araguaia apresentou o maior número de espécies exclusivas (21), seguido do Parque Nacional das Nascentes do Rio Parnaíba (18), Parque Nacional Grande Sertão Veredas (11), e Estação Ecológica Serra Geral do Tocantins e Refúgio de Vida Silvestre das Veredas do Oeste Baiano (7). A fauna dos pontos amostrados no Parque Nacional Grande Sertão Veredas foi a mais homogênea em termos de composição de espécies (Stress = 19.1, Anosim total $\mathrm{R}=0.40, \mathrm{p}=0.001$, veja Tab. IV para comparações par a par) (Fig. 2). Apesar dos pontos das demais UCs mostrarem um padrão consideravelmente dissimilar entre si (Fig. 2), a análise de similaridade mostrou que a composição de espécies difere entre as UCs (exceto para PNNRP x EESG, Tab. IV).

Fitofisionomias. Das seis fitofisionomias amostradas, campo cerrado foi a que apresentou maior abundância de Arctiinae, seguido por cerrado sensu stricto, cerradão, mata semidecídua, campo sujo e vereda (Tab. V). O mesmo padrão foi observado para a riqueza de espécies (Tab. VI). Contudo, quando a riqueza foi padronizada pelo número de indivíduos, o cerradão e a mata semidecídua apresentaram uma fauna significativamente mais rica do que o campo cerrado e cerrado sensu stricto (Fig. 3).

Cinquenta e quatro espécies ocorreram em uma única fitofisionomia e nenhuma ocorreu em todas as fitofisionomias. A mata semidecídua e o cerradão revelaram o maior número de espécies exclusivas (ambos com 14), seguido do campo cerrado (12), cerrado sensu stricto (10), 
Tab. I. Descrição das cinco Unidades de Conservação (UC) amostradas (MAT, mata; CSS, cerrado sensu stricto; CC, campo cerrado; CER, cerradão; CS, campo sujo; VER, vereda).

\begin{tabular}{|c|c|c|c|c|c|c|}
\hline $\mathrm{UC}$ & Estado & $\begin{array}{l}\text { Município mais próximo } \\
\text { da área de coleta }\end{array}$ & Coordenadas & Datas das coletas & $\begin{array}{c}\text { Fitofisionomias } \\
\text { amostradas }\end{array}$ & $\begin{array}{c}\text { Estado de } \\
\text { conservação da } \\
\text { vegetação }\end{array}$ \\
\hline $\begin{array}{l}\text { Parque Estadual do } \\
\text { Araguaia }\end{array}$ & $\begin{array}{l}\text { Mato } \\
\text { Grosso }\end{array}$ & Novo Santo Antônio & $\begin{array}{l}12^{\circ} 22^{\prime} 13,8^{\prime \prime} \mathrm{S} \\
50^{\circ} 56^{\prime} 42,2^{\prime \prime} \mathrm{N}\end{array}$ & 25 a $28 / 01 / 2012$ & CER, MAT & $\begin{array}{l}\text { Alterado } \\
\text { (gado) }\end{array}$ \\
\hline $\begin{array}{l}\text { Parque Nacional das } \\
\text { Nascentes do Rio } \\
\text { Parnaíba }\end{array}$ & Piauí & Barreiras do Piauí & $\begin{array}{l}10^{\circ} 02{ }^{\prime} 10,1{ }^{\prime \prime} \mathrm{S} \\
45^{\circ} 40^{\prime} 53,33^{\prime \prime} \mathrm{N}\end{array}$ & 17 a $20 / 02 / 2012$ & $\begin{array}{l}\text { CER, CSS, } \\
\text { VER, CC }\end{array}$ & Preservado \\
\hline $\begin{array}{l}\text { Parque Nacional } \\
\text { Grande Sertão } \\
\text { Veredas }\end{array}$ & $\begin{array}{l}\text { Minas } \\
\text { Gerais }\end{array}$ & Chapada Gaúcha & $\begin{array}{l}15^{\circ} 03^{\prime} 23^{\prime \prime} \mathrm{S} ; \\
45^{\circ} 48^{\prime} 09^{\prime \prime} \mathrm{N}\end{array}$ & 18 a $21 / 02 / 2013$ & $\mathrm{CC}, \mathrm{CSS}$ & Preservado \\
\hline $\begin{array}{l}\text { Estação Ecológica } \\
\text { Serra Geral do } \\
\text { Tocantins }\end{array}$ & Tocantins & Dianópolis & $\begin{array}{l}11^{\circ} 17^{\prime} 15,22^{\prime} \mathrm{S} ; \\
46^{\circ} 57^{\prime} 34,0^{\prime \prime} \mathrm{N}\end{array}$ & 24 a $27 / 02 / 2012$ & $\mathrm{CC}, \mathrm{CS}$ & Preservado \\
\hline $\begin{array}{l}\text { Refúgio de Vida } \\
\text { Silvestre das Veredas } \\
\text { do Oeste Baiano }\end{array}$ & Bahia & Cocos & $\begin{array}{l}14^{\circ} 13^{\prime} 07,5^{\prime \prime} \mathrm{S} \\
45^{\circ} 38^{\prime} 45,0^{\prime \prime} \mathrm{N}\end{array}$ & 04 a 07/02/2013 & $\mathrm{CC}, \mathrm{CSS}$ & $\begin{array}{l}\text { Alterado } \\
\text { (gado, } \\
\text { monocultura) }\end{array}$ \\
\hline
\end{tabular}

Tab. II. Abundância das espécies de mariposas Arctiinae (Lepidoptera, Erebidae) nas cinco Unidades de Conservação de Cerrado amostradas (PNN, Parque Nacional das Nascentes do Rio Parnaíba; PE, Parque Estadual do Araguaia; EESG, Estação Ecológica Serra Geral do Tocantins; RVSV, Refúgio de Vida Silvestre das Veredas do Oeste Baiano; PNGS, Parque Nacional Grande Sertão Veredas).

\begin{tabular}{|c|c|c|c|c|c|c|}
\hline & PNN & $\mathrm{PE}$ & EESG & RVSV & PNGS & Total \\
\hline Achroosia nuda Hampson, 1900 & 2 & 0 & 0 & 0 & 0 & 2 \\
\hline Aclytia heber (Cramer, 1780) & 0 & 12 & 0 & 4 & 1 & 17 \\
\hline Aclytia sp. 1 & 0 & 0 & 0 & 0 & 1 & 1 \\
\hline Aethria haemorrhoidalis (Stoll, 1790) & 0 & 1 & 0 & 0 & 0 & 1 \\
\hline Agaraea minuta Schaus, 1892 & 0 & 1 & 0 & 0 & 0 & 1 \\
\hline Agaraea semivitrea Rothschild, 1909 & 0 & 1 & 0 & 1 & 0 & 2 \\
\hline Amaxia kennedyi (Rothschild, 1909) & 0 & 0 & 1 & 0 & 0 & 1 \\
\hline Ammalo sp.1 & 1 & 0 & 0 & 0 & 0 & 1 \\
\hline Arctiini sp.4 & 0 & 0 & 0 & 1 & 0 & 1 \\
\hline Calodesma collaris (Drury, 1782) & 0 & 1 & 0 & 0 & 0 & 1 \\
\hline Cercocladia novicia Draudt, 1915 & 3 & 0 & 0 & 0 & 0 & 3 \\
\hline Chrysostola nivaca (Jones, 1914) & 46 & 1 & 2 & 5 & 156 & 210 \\
\hline Cisthene griseola (Rothschild, 1913) & 1 & 0 & 0 & 0 & 0 & 1 \\
\hline Cisthene subrubra (Schaus, 1905) & 0 & 0 & 0 & 0 & 10 & 10 \\
\hline Cisthene triplaga Hampson, 1905 & 1 & 0 & 0 & 1 & 2 & 4 \\
\hline Cisthene xanthospila (Hampson, 1900) & 1 & 0 & 0 & 0 & 0 & 1 \\
\hline Correbidia sp. 2 & 0 & 1 & 0 & 0 & 0 & 1 \\
\hline Cosmosoma achemon (Fabricius, 1781) & 0 & 0 & 0 & 1 & 0 & 1 \\
\hline Cosmosoma rasera Jones, 1914 & 1 & 0 & 0 & 0 & 0 & 1 \\
\hline Cosmosoma restrictum Butler, 1876 & 0 & 1 & 0 & 0 & 0 & 1 \\
\hline Delphyre discalis (Druce, 1905) & 0 & 1 & 0 & 0 & 0 & 1 \\
\hline Delphyre hebes Walker, 1854 & 0 & 2 & 0 & 0 & 0 & 2 \\
\hline Disconeura lutosa (Hübner, [1823]) & 0 & 5 & 0 & 0 & 0 & 5 \\
\hline Dycladia lucetius (Cramer, 1782) & 1 & 1 & 1 & 3 & 5 & 11 \\
\hline Dysschema sacrifica (Hübner, [1831]) & 0 & 0 & 1 & 0 & 0 & 1 \\
\hline Episcepsis thetis (Linnaeus, 1771) & 0 & 1 & 0 & 0 & 0 & 1 \\
\hline Eupseudosoma grandis Rothschild, 1909 & 0 & 0 & 0 & 0 & 1 & 1 \\
\hline Eucereon cineta Schaus, 1896 & 0 & 1 & 0 & 0 & 0 & 1 \\
\hline Eucereon sylvius (Stoll, 1790) & 0 & 1 & 0 & 0 & 0 & 1 \\
\hline Euchaetes sp.1 & 1 & 0 & 2 & 0 & 0 & 3 \\
\hline Eupseudosoma larissa (Druce, 1890) & 0 & 1 & 0 & 0 & 0 & 1 \\
\hline Heliura tetragramma (Walker, 1854) & 4 & 0 & 0 & 3 & 60 & 67 \\
\hline Hyperthaema sp.1 & 0 & 0 & 0 & 1 & 0 & 1 \\
\hline Hyda basilutea (Walker, 1854) & 1 & 0 & 0 & 0 & 0 & 1 \\
\hline Hyperandra appendiculata (Herrich-Schäffer, [1856]) & 0 & 1 & 0 & 0 & 0 & 1 \\
\hline Hypercompe sp.1 & 0 & 1 & 0 & 0 & 0 & 1 \\
\hline Hypocrita sp. 1 & 0 & 1 & 0 & 0 & 0 & 1 \\
\hline Ichoria chalcomedusa Druce, 1893 & 1 & 0 & 0 & 0 & 0 & 1 \\
\hline Idalus carinosa (Schaus, 1905) & 17 & 1 & 6 & 6 & 11 & 41 \\
\hline
\end{tabular}


Tab. II. Cont.

\begin{tabular}{|c|c|c|c|c|c|c|}
\hline & PNN & $\mathrm{PE}$ & EESG & RVSV & PNGS & Total \\
\hline Idalus lineosus Walker, 1869 & 0 & 2 & 0 & 0 & 0 & 2 \\
\hline Idalus vitrea (Cramer, 19780) & 0 & 0 & 1 & 0 & 0 & 1 \\
\hline Isia alcumena (Berg, 1882) & 0 & 0 & 1 & 0 & 0 & 1 \\
\hline Lepidokirbyia vittipes (Schaus, 1896) & 0 & 0 & 0 & 0 & 2 & 2 \\
\hline Lepidoneiva erubescens (Butler, 1876) & 0 & 0 & 1 & 0 & 0 & 1 \\
\hline Leucanopsis sp.1 & 0 & 2 & 0 & 0 & 0 & 2 \\
\hline Leucanopsis sp.3 & 1 & 0 & 0 & 0 & 0 & 1 \\
\hline Lithosiina sp.1 & 0 & 0 & 0 & 3 & 0 & 3 \\
\hline Lithosiina sp.2 & 0 & 0 & 0 & 2 & 2 & 4 \\
\hline Lophocampa citrina (Sepp, [1852]) & 0 & 0 & 0 & 0 & 4 & 4 \\
\hline Lophocampa pectina (Schaus, 1896) & 0 & 0 & 0 & 1 & 3 & 4 \\
\hline Lophocampa $\mathrm{sp} .1$ & 1 & 0 & 0 & 0 & 0 & 1 \\
\hline Macrocneme aurifera Hampson, 1914 & 0 & 0 & 1 & 0 & 1 & 2 \\
\hline Macrocneme sp.1 & 0 & 2 & 0 & 0 & 0 & 2 \\
\hline Melese incertus (Walker, 1855) & 1 & 0 & 0 & 0 & 0 & 1 \\
\hline Napata terminalis (Walker, 1854) & 0 & 2 & 0 & 0 & 0 & 2 \\
\hline Neritos atta Schaus, 1920 & 0 & 2 & 8 & 0 & 36 & 46 \\
\hline Nodozana jucunda Jones, 1914 & 0 & 0 & 0 & 2 & 3 & 5 \\
\hline Odozana obscura (Schaus, 1896) & 0 & 0 & 3 & 7 & 0 & 10 \\
\hline Odozana sp.1 & 0 & 0 & 0 & 0 & 10 & 10 \\
\hline Paracles sp.1 & 1 & 0 & 0 & 0 & 0 & 1 \\
\hline Pareuchaetes aurata (Butler, 1875) & 0 & 0 & 1 & 0 & 3 & 4 \\
\hline Pheia albisigna (Walker, 1854) & 0 & 0 & 0 & 0 & 1 & 1 \\
\hline Pheia haemopera Schaus, 1898 & 1 & 0 & 0 & 0 & 0 & 1 \\
\hline Pheia haematosticta Jones, 1908 & 0 & 0 & 0 & 0 & 2 & 2 \\
\hline Pheia seraphina (Herrich-Schäffer, 1854) & 0 & 0 & 0 & 0 & 2 & 2 \\
\hline Philoros rubriceps (Walker, 1854) & 8 & 0 & 5 & 2 & 12 & 27 \\
\hline Phoenicoprocta baeri Rothschild, 1911 & 0 & 0 & 0 & 0 & 1 & 1 \\
\hline Phoenicoprocta haemorrhoidalis (Fabricius, 1775) & 0 & 0 & 0 & 0 & 1 & 1 \\
\hline Phoenicoprocta ignicauda Draudt, 1915 & 2 & 0 & 0 & 0 & 0 & 2 \\
\hline Phoenicoprocta rubiventer Hampson, 1898 & 0 & 0 & 0 & 1 & 6 & 7 \\
\hline Phoenicoprocta sp.1 & 9 & 0 & 0 & 0 & 0 & 9 \\
\hline Pryteria alboatra (Rothschild, 1909) & 0 & 1 & 0 & 1 & 1 & 3 \\
\hline Pseudalus limona Schaus, 1896 & 0 & 0 & 1 & 0 & 0 & 1 \\
\hline Pseudalus salmonaceus (Rothschild, 1909) & 0 & 0 & 0 & 1 & 0 & 1 \\
\hline Pseudosphex aequalis (Walker, 1864) & 2 & 0 & 0 & 0 & 0 & 2 \\
\hline Pseudosphex sodalis Draudt, 1905 & 13 & 0 & 18 & 0 & 1 & 32 \\
\hline Psilopleura pentheri Zerny, 1912 & 0 & 2 & 0 & 0 & 0 & 2 \\
\hline Sciopsyche tropica (Walker, 1854) & 1 & 0 & 0 & 0 & 0 & 1 \\
\hline Trichromia sp. 1 & 0 & 0 & 0 & 1 & 0 & 1 \\
\hline Trichromia sp.2 & 0 & 4 & 0 & 0 & 0 & 4 \\
\hline Utetheisa ornatrix (Linnaeus, 1758) & 0 & 0 & 2 & 0 & 0 & 2 \\
\hline Viviennea salma (Druce, 1896) & 0 & 0 & 0 & 1 & 0 & 1 \\
\hline Zatrephes fasciola Seitz, 1922 & 1 & 0 & 0 & 0 & 0 & 1 \\
\hline Abundância total & 122 & 53 & 55 & 48 & 338 & 616 \\
\hline
\end{tabular}

Tab. III. Riqueza observada (Riq. Obs.) e estimada (Jackknife de primeira ordem \pm desvio padrão, Jack1 \pm DV e Chao de segunda ordem, Chao $2 \pm$ DV) e porcentagem da riqueza estimada que foi amostrada (\% amostrada) de mariposas Arctiinae (Lepidoptera, Erebidae) nas cinco Unidades de Conservação amostradas (PNN, Parque Nacional das Nascentes do Rio Parnaíba; PE, Parque Estadual do Araguaia; EESG, Estação Ecológica Serra Geral do Tocantins; RVSV, Refúgio de Vida Silvestre das Veredas do Oeste Baiano; PNGS, Parque Nacional Grande Sertão Veredas).

\begin{tabular}{lcccc}
\hline & PNN & PE & EESG & RVSV \\
\hline Riq. Obs. & 33 & 30 & 17 & 22 \\
Jack1 \pm DV & $43,9 \pm 8,4$ & $49,5 \pm 9,0$ & $26,09 \pm 3,7$ & $32,9 \pm 4,07$ \\
Chao 2 \pm DV & $50,95 \pm 14,8$ & $68,06 \pm 4,5$ & $30,5 \pm 10,21$ & $35,3 \pm 10,7$ \\
$\%$ amostrada Jack & 75 & 60 & 65 & 67 \\
$\%$ amostrada Chao & 64 & 44 & 56 & 62 \\
\hline
\end{tabular}

campo sujo (3) e vereda (1). De todas as fitofisionomias amostradas, a fauna dos pontos de mata semidecídua foi a que mostrou a menor dissimilaridade $($ Stress $=18.9$, Anosim total $\mathrm{R}=0.14, \mathrm{p}=0.01$, veja Tab. VII para comparações par a par) (Fig. 4). A mata semidecídua foi dissimilar de campo cerrado e de cerrado sensu stricto, e as demais fitofisionomias não diferiram na composição de espécies (Tab. VII). As fitofisionomias foram mais importantes do que as Unidades de Conservação em explicar a diferença na riqueza de Arctiinae (Tab. VIII). 


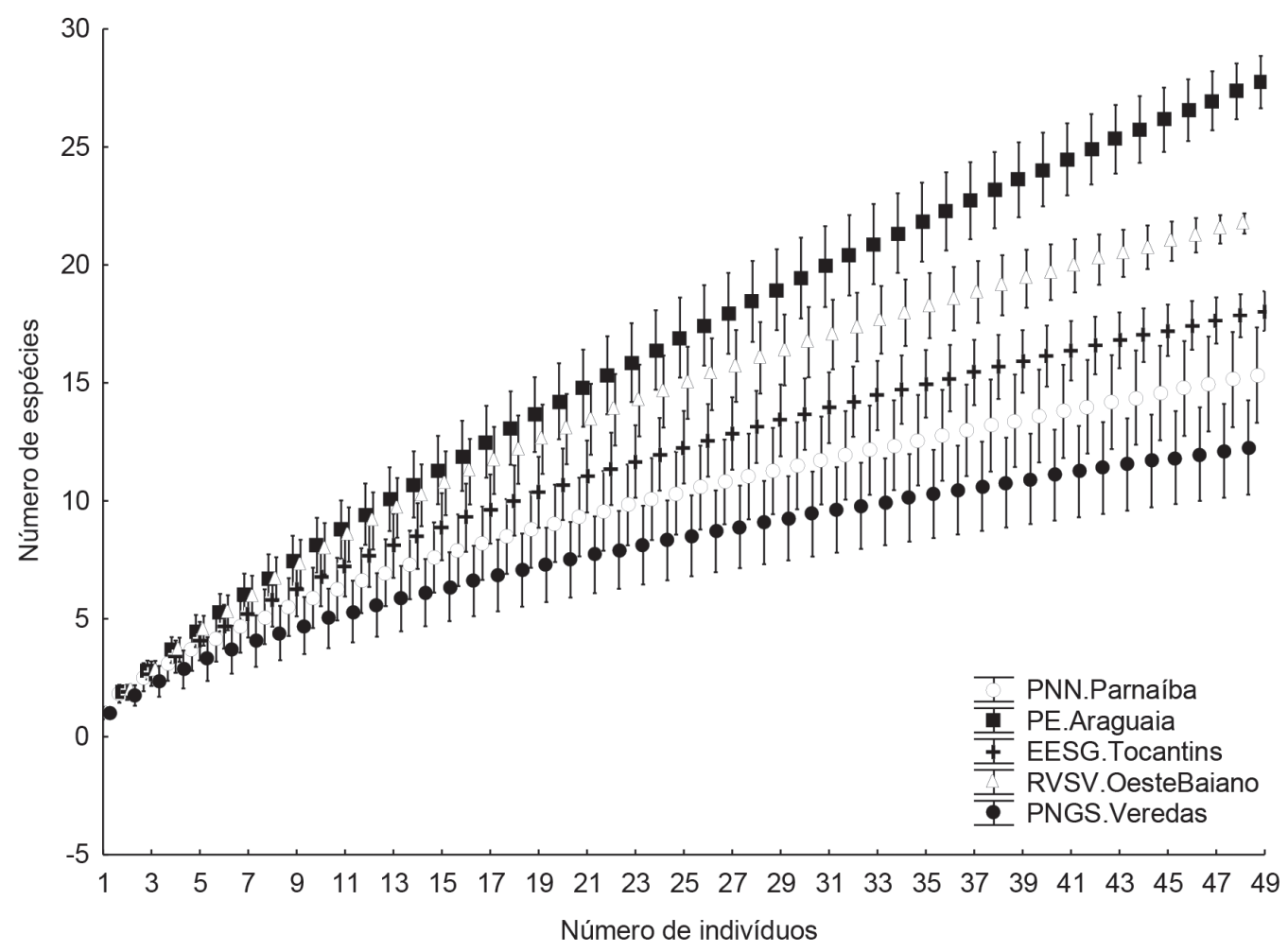

Fig. 1. Riqueza rarefeita da fauna de Arctiinae (Lepidoptera, Erebidae) em cinco Unidades de Conservação de Cerrado. As barras representam o intervalo de confiança (PNN. Parnaíba, Parque Nacional das Nascentes do Rio Parnaíba; PE.Araguaia, Parque Estadual do Araguaia; EESG.Tocantins, Estação Ecológica Serra Geral do Tocantins; RVSV.Oeste Baiano, Refúgio de Vida Silvestre das Veredas do Oeste Baiano; PNGS.Veredas, Parque Nacional Grande Sertão Veredas).

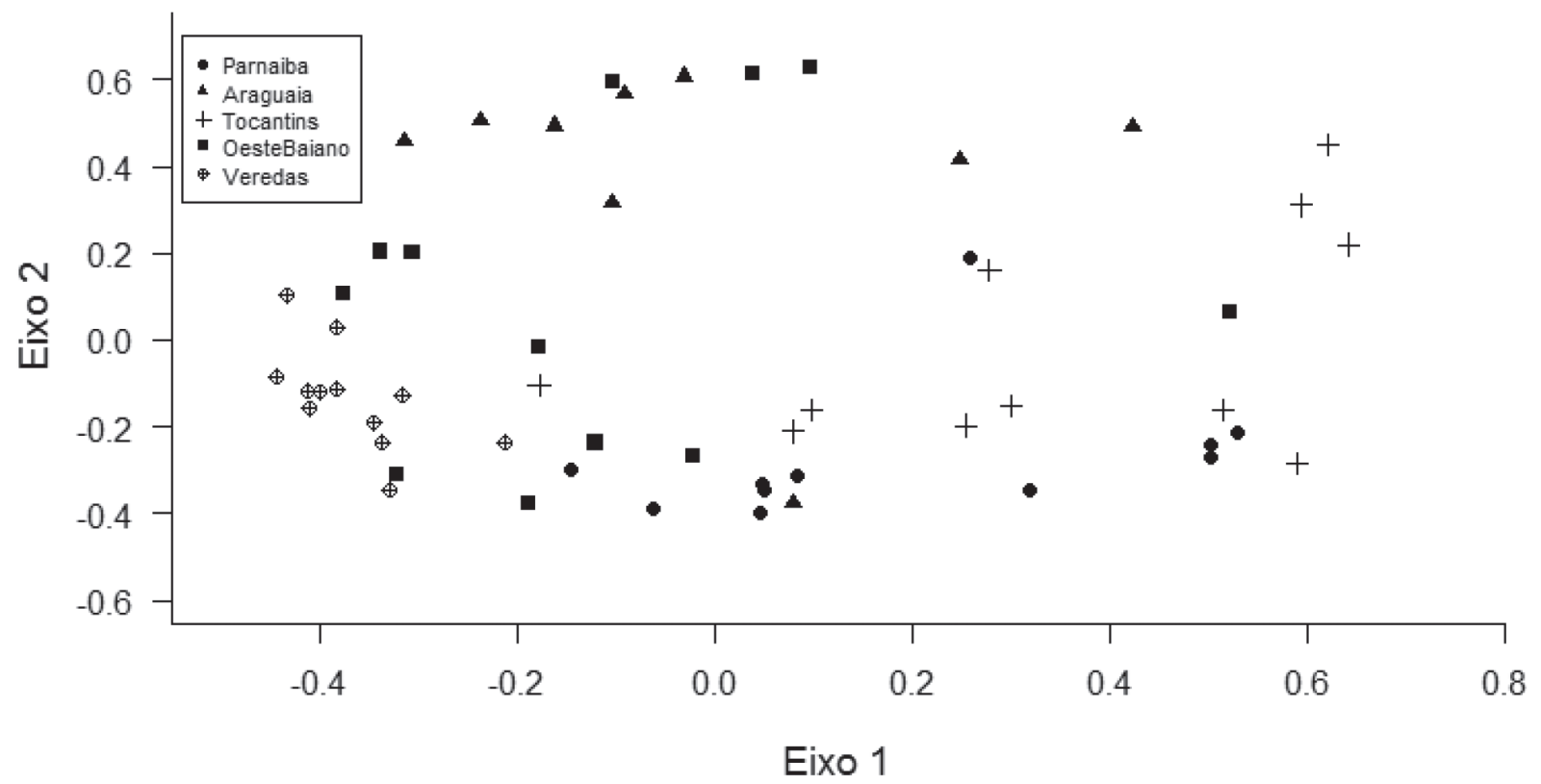

Fig. 2. Análise NMDS (Stress $=19.1$ ) realizada para fauna de Arctiinae (Lepidoptera, Erebidae) amostrada em cinco Unidades de Conservação localizadas no Cerrado (Parnaíba, Parque Nacional das Nascentes do Rio Parnaíba; Araguaia, Parque Estadual do Araguaia; Tocantins, Estação Ecológica Serra Geral do Tocantins; Oeste Baiano, Refúgio de Vida Silvestre das Veredas do Oeste Baiano; Veredas, Parque Nacional Grande Sertão Veredas). 


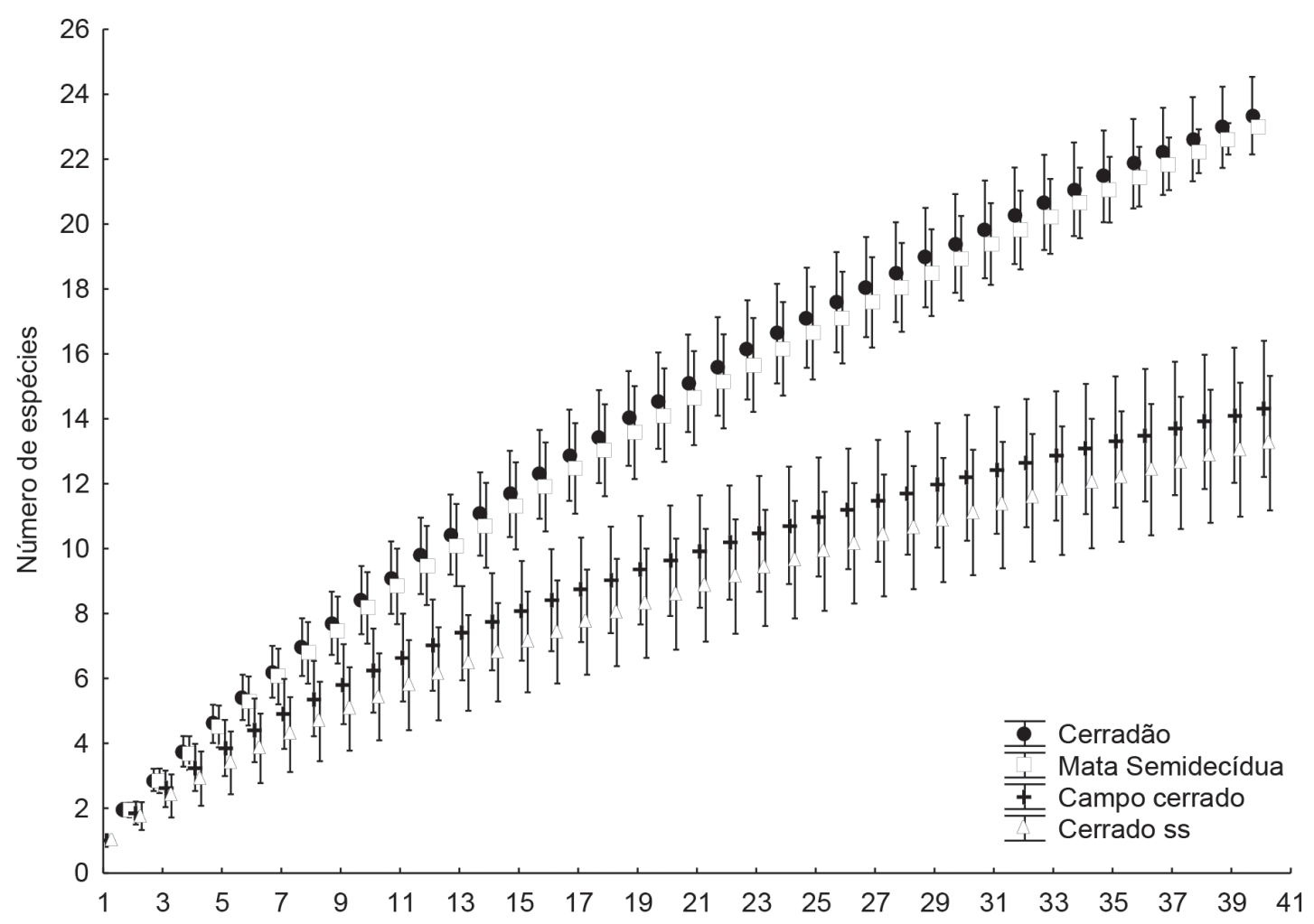

Fig. 3. Riqueza rarefeita da fauna de Arctiinae (Lepidoptera, Erebidae) em quatro fitofisionomias de Cerrado. Campo sujo e vereda não foram incluídas na análise devido à baixa quantidade de amostras. As barras representam o intervalo de confiança. Cerrado ss indica cerrado sensu stricto.

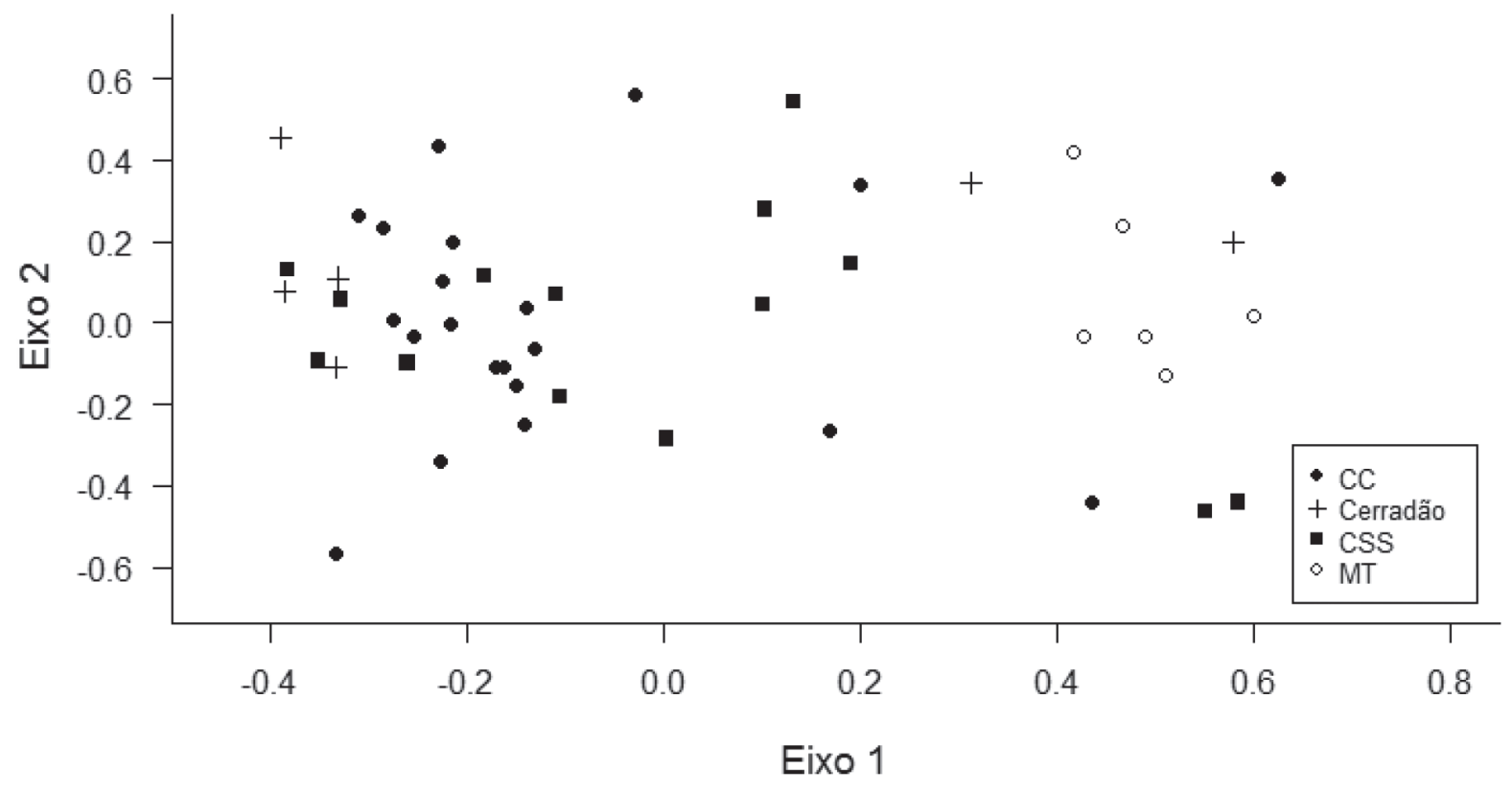

Fig. 4. Análise de NMDS (Stress = 18.9) realizada para fauna de Arctiinae (Lepidoptera, Erebidae) amostrada em quatro fitofisionomias de Cerrado (CC, campo cerrado; CSS, cerrado sensu stricto; MT, mata estacional semidecídua). Campo sujo e vereda não foram incluídas na análise devido à baixa quantidade de amostras. 
Tab. IV. Análise de similaridade (ANOSIM) par a par, entre as Unidades de Conservação (UCs) com os valores da estatística R, p e a correção de Bonferroni (para múltiplas comparações, usado para reduzir erro tipo 1) (PEA, Parque Estadual do Araguaia; RVSV, Refúgio de Vida Silvestre do Oeste Baiano; PNGSV, Parque Nacional Grande Sertão Veredas; PNNRP, Parque Nacional das Nascentes do Rio Parnaíba; EESG, Estação Ecológica Serra Geral do Tocantins).

\begin{tabular}{lccc}
\hline UCs & $\mathrm{R}$ & $\mathrm{p}$ & $\mathrm{p}$ de Bonferroni \\
\hline PEA x RVSV & 0,21 & $0,006^{*}$ & $0,001^{*}$ \\
PEA x PNGSV & 0,76 & $0,001^{*}$ & $024^{*}$ \\
PEA x PNNRP & 0,6 & $0,001^{*}$ & $0,04^{*}$ \\
PEA x EESG & 0,41 & $0,001^{*}$ \\
RVSV x PNGSV & 0,45 & $0,001^{*}$ \\
RVSV x PNNRP & 0,28 & $0,004^{*}$ \\
RVSV x EESG & 0,23 & $0,002^{*}$ & $0,004^{*}$ \\
PNGSV x PNNRP & 0,59 & $0,001 *$ & $0,004^{*}$ \\
PNGSV x EESG & 0,54 & $0,001^{*}$ & $0,004 *$ \\
PNNRP x EESG & 0,04 & 0,19 & $0,004 *$ \\
\hline
\end{tabular}

Tab. V. Abundância das espécies de mariposas Arctiinae (Lepidoptera, Erebidae) nas seis fitofisionomias amostradas no estudo (CER, cerradão; MAT, mata semidecídua; CS, campo sujo; VER, vereda; CC, campo cerrado; CSS, cerrado sensu stricto).

\begin{tabular}{|c|c|c|c|c|c|c|}
\hline & CER & MAT & $\mathrm{CS}$ & VER & $\mathrm{CC}$ & CSS \\
\hline Achroosia nuda Hampson, 1900 & 2 & 0 & 0 & 0 & 0 & 0 \\
\hline Aclytia heber (Cramer, 1780) & 3 & 8 & 0 & 0 & 2 & 4 \\
\hline Aclytia sp. 1 & 0 & 0 & 0 & 0 & 0 & 1 \\
\hline Aethria haemorrhoidalis (Stoll, 1790) & 0 & 1 & 0 & 0 & 0 & 0 \\
\hline Agaraea minuta Schaus, 1892 & 0 & 1 & 0 & 0 & 0 & 0 \\
\hline Agaraea semivitrea Rothschild, 1909 & 0 & 1 & 0 & 0 & 1 & 0 \\
\hline Amaxia kennedyi (Rothschild, 1909) & 0 & 0 & 1 & 0 & 0 & 0 \\
\hline Ammalo sp.1 & 0 & 0 & 0 & 1 & 0 & 0 \\
\hline Arctiini sp. 4 & 0 & 0 & 0 & 0 & 0 & 1 \\
\hline Calodesma collaris (Drury, 1782) & 0 & 1 & 0 & 0 & 0 & 0 \\
\hline Cercocladia novicia Draudt, 1915 & 0 & 0 & 0 & 0 & 3 & 0 \\
\hline Chrysostola nivaca (Jones, 1914) & 8 & 0 & 0 & 0 & 93 & 109 \\
\hline Cisthene griseola (Rothschild, 1913) & 1 & 0 & 0 & 0 & 0 & 0 \\
\hline Cisthene subrubra (Schaus, 1905) & 0 & 0 & 0 & 0 & 1 & 9 \\
\hline Cisthene triplaga Hampson, 1905 & 2 & 0 & 0 & 0 & 1 & 1 \\
\hline Cisthene xanthospila (Hampson, 1900) & 1 & 0 & 0 & 0 & 0 & 0 \\
\hline Correbidia sp. 2 & 0 & 1 & 0 & 0 & 0 & 0 \\
\hline Cosmosoma achemon (Fabricius, 1781) & 0 & 0 & 0 & 0 & 1 & 0 \\
\hline Cosmosoma rasera Jones, 1914 & 1 & 0 & 0 & 0 & 0 & 0 \\
\hline Cosmosoma restrictum Butler, 1876 & 0 & 1 & 0 & 0 & 0 & 0 \\
\hline Delphyre discalis (Druce, 1905) & 1 & 0 & 0 & 0 & 0 & 0 \\
\hline Delphyre hebes Walker, 1854 & 0 & 2 & 0 & 0 & 0 & 0 \\
\hline Disconeura lutosa (Hübner, [1823]) & 1 & 4 & 0 & 0 & 0 & 0 \\
\hline Dycladia lucetius (Cramer, 1782) & 0 & 1 & 0 & 0 & 7 & 3 \\
\hline Dysschema sacrifica (Hübner, [1831]) & 0 & 0 & 0 & 0 & 1 & 0 \\
\hline Episcepsis thetis (Linnaeus, 1771) & 0 & 1 & 0 & 0 & 0 & 0 \\
\hline Eupseudosoma grandis Rothschild, 1909 & 0 & 0 & 0 & 0 & 0 & 1 \\
\hline Eucereon cineta Schaus, 1896 & 0 & 1 & 0 & 0 & 0 & 0 \\
\hline Eucereon sylvius (Stoll, 1790) & 0 & 1 & 0 & 0 & 0 & 0 \\
\hline Euchaetes sp. 1 & 0 & 0 & 1 & 1 & 1 & 0 \\
\hline Eupseudosoma larissa (Druce, 1890) & 1 & 0 & 0 & 0 & 0 & 0 \\
\hline Heliura tetragramma (Walker, 1854) & 1 & 0 & 0 & 0 & 43 & 23 \\
\hline Hyperthaema sp. 1 & 0 & 0 & 0 & 0 & 0 & 1 \\
\hline Hyda basilutea (Walker, 1854) & 0 & 0 & 0 & 0 & 1 & 0 \\
\hline Hyperandra appendiculata (Herrich-Schäffer, [1856]) & 0 & 1 & 0 & 0 & 0 & 0 \\
\hline Hypercompe sp. 1 & 0 & 1 & 0 & 0 & 0 & 0 \\
\hline Hypocrita sp. 1 & 0 & 1 & 0 & 0 & 0 & 0 \\
\hline Ichoria chalcomedusa Druce, 1893 & 1 & 0 & 0 & 0 & 0 & 0 \\
\hline Idalus carinosa (Schaus, 1905) & 4 & 0 & 1 & 0 & 30 & 6 \\
\hline Idalus lineosus Walker, 1869 & 2 & 0 & 0 & 0 & 0 & 0 \\
\hline Idalus vitrea (Cramer, 19780) & 0 & 0 & 0 & 0 & 1 & 0 \\
\hline Isia alcumena (Berg, 1882) & 0 & 0 & 1 & 0 & 0 & 0 \\
\hline Lepidokirbyia vittipes (Schaus, 1896) & 0 & 0 & 0 & 0 & 0 & 2 \\
\hline Lepidoneiva erubescens (Butler, 1876) & 0 & 0 & 0 & 0 & 1 & 0 \\
\hline Leucanopsis sp. 1 & 0 & 2 & 0 & 0 & 0 & 0 \\
\hline Leucanopsis sp. 3 & 1 & 0 & 0 & 0 & 0 & 0 \\
\hline Lithosiina sp. 1 & 0 & 0 & 0 & 0 & 1 & 2 \\
\hline Lithosiina sp. 2 & 0 & 0 & 0 & 0 & 3 & 1 \\
\hline
\end{tabular}


Tab. V. Cont.

\begin{tabular}{|c|c|c|c|c|c|c|}
\hline & CER & MAT & $\mathrm{CS}$ & VER & $\mathrm{CC}$ & CSS \\
\hline Lophocampa citrina (Sepp, [1852]) & 0 & 0 & 0 & 0 & 3 & 1 \\
\hline Lophocampa pectina (Schaus, 1896) & 1 & 0 & 0 & 0 & 3 & 0 \\
\hline Lophocampa sp. 1 & 1 & 0 & 0 & 0 & 0 & 0 \\
\hline Macrocneme aurifera Hampson, 1914 & 0 & 0 & 0 & 0 & 1 & 1 \\
\hline Macrocneme sp. 1 & 2 & 0 & 0 & 0 & 0 & 0 \\
\hline Melese incertus (Walker, 1855) & 0 & 0 & 0 & 0 & 1 & 0 \\
\hline Napata terminalis (Walker, 1854) & 0 & 2 & 0 & 0 & 0 & 0 \\
\hline Neritos atta Schaus, 1920 & 0 & 2 & 1 & 0 & 20 & 23 \\
\hline Nodozana jucunda Jones, 1914 & 0 & 0 & 0 & 0 & 1 & 4 \\
\hline Odozana obscura (Schaus, 1896) & 0 & 0 & 0 & 0 & 1 & 9 \\
\hline Odozana sp. 1 & 0 & 0 & 0 & 0 & 8 & 2 \\
\hline Paracles sp. 1 & 0 & 0 & 0 & 0 & 0 & 1 \\
\hline Pareuchaetes aurata (Butler, 1875) & 0 & 0 & 1 & 0 & 2 & 1 \\
\hline Pheia albisigna (Walker, 1854) & 0 & 0 & 0 & 0 & 0 & 1 \\
\hline Pheia haemopera Schaus, 1898 & 0 & 0 & 0 & 0 & 1 & 0 \\
\hline Pheia haematosticta Jones, 1908 & 0 & 0 & 0 & 0 & 1 & 1 \\
\hline Pheia seraphina (Herrich-Schäffer, 1854) & 0 & 0 & 0 & 0 & 1 & 1 \\
\hline Philoros rubriceps (Walker, 1854) & 3 & 0 & 1 & 0 & 16 & 7 \\
\hline Phoenicoprocta baeri Rothschild, 1911 & 0 & 0 & 0 & 0 & 0 & 1 \\
\hline Phoenicoprocta haemorrhoidalis (Fabricius, 1775) & 0 & 0 & 0 & 0 & 0 & 1 \\
\hline Phoenicoprocta ignicauda Draudt, 1915 & 1 & 0 & 1 & 0 & 0 & 0 \\
\hline Phoenicoprocta rubiventer Hampson, 1898 & 0 & 0 & 0 & 0 & 4 & 3 \\
\hline Phoenicoprocta sp. 1 & 2 & 0 & 0 & 0 & 7 & 0 \\
\hline Pryteria alboatra (Rothschild, 1909) & 0 & 1 & 0 & 0 & 0 & 2 \\
\hline Pseudalus limona Schaus, 1896 & 0 & 0 & 1 & 0 & 0 & 0 \\
\hline Pseudalus salmonaceus (Rothschild, 1909) & 0 & 0 & 0 & 0 & 1 & 0 \\
\hline Pseudosphex aequalis (Walker, 1864) & 2 & 0 & 0 & 0 & 0 & 0 \\
\hline Pseudosphex sodalis Draudt, 1905 & 3 & 0 & 3 & 2 & 19 & 5 \\
\hline Psilopleura pentheri Zerny, 1912 & 2 & 0 & 0 & 0 & 0 & 0 \\
\hline Sciopsyche tropica (Walker, 1854) & 1 & 0 & 0 & 0 & 0 & 0 \\
\hline Trichromia sp. 1 & 0 & 0 & 0 & 0 & 0 & 1 \\
\hline Trichromia sp. 2 & 1 & 3 & 0 & 0 & 0 & 0 \\
\hline Utetheisa ornatrix (Linnaeus, 1758) & 0 & 0 & 0 & 0 & 2 & 0 \\
\hline Viviennea salma (Druce, 1896) & 0 & 0 & 0 & 0 & 1 & 0 \\
\hline Zatrephes fasciola Seitz, 1922 & 0 & 0 & 0 & 0 & 1 & 0 \\
\hline Abundância total & 49 & 37 & 12 & 4 & 285 & 229 \\
\hline
\end{tabular}

Tab. VI. Riqueza observada (Riq. Obs.) e estimada (Jackknife de primeira ordem \pm desvio padrão, Jack1 \pm DV e Chao de segunda ordem, Chao $2 \pm$ DV) e porcentagem da riqueza estimada que foi amostrada (\% amostrada) de mariposas Arctiinae (Lepidoptera, Erebidae) nas seis fitofisionomias amostradas (MAT, mata semidecídua; CER, cerradão; CSS, cerrado sensu stricto; CC, campo cerrado; CS, campo sujo; VER, vereda).

\begin{tabular}{lcccccc}
\hline & MAT & CER & CSS & CC & CS & VER \\
\hline Riq. Obs. & 23 & 25 & 32 & 39 & 10 & 3 \\
Jack1 \pm DV & $36,3 \pm 7,2$ & $37,5 \pm 5,9$ & $49,7 \pm 4,1$ & $59 \pm 6,17$ & $17,2 \pm 3,7$ & $4,5 \pm 1,3$ \\
Chao2 \pm DV & $55,8 \pm 21,4$ & $37,5 \pm 8,7$ & $56,6 \pm 15,2$ & $67,7 \pm 5,8$ & $24,4 \pm 13$ & $4,5 \pm 2,25$ \\
$\%$ amostrada Jack & 63 & 66 & 64 & 66 & 58 & 66 \\
$\%$ amostrada Chao & 41 & 66 & 56 & 57 & 40 & 66 \\
\hline
\end{tabular}

Tab. VII. Análise de similaridade (ANOSIM) par a par, entre as fitofisionomias com os valores da estatística R, p e a correção de Bonferroni (para múltiplas comparações, usado para reduzir erro tipo 1). Cer significa cerradão, Mat mata semidecídua, CC campo cerrado e CSS cerrado sensu stricto.

\begin{tabular}{lccc}
\hline Fitofisionomias & $\mathrm{R}$ & $\mathrm{p}$ & $\mathrm{p}$ de Bonferroni \\
\hline Cer X Mat & 0,27 & $0,04^{*}$ & 0,12 \\
CC X Mat & 0,41 & $0,001^{*}$ & $0,003^{*}$ \\
CSS X Mat & 0,37 & $0,004^{*}$ & $0,012^{*}$ \\
Cer X CC & 0,02 & 0,33 & 0,99 \\
Cer x CSS & 0,13 & 0,11 & 0,31 \\
CC x CSS & 0,01 & 0,33 & 0,99 \\
\hline
\end{tabular}

Tab. VIII. Resultados da análise de GLM (Modelo Linear Generalizado) predizendo a variável resposta (riqueza de mariposas Arctiinae) por duas variáveis categóricas (Unidades de Conservação e fitofisionomias). O modelo mais representativo foi escolhido através dos valores estatísticos de AICc.

\begin{tabular}{lcc}
\hline Modelo & GL & AICc \\
\hline Nulo & 1 & 75,86948 \\
Fitofisionomias & 4 & 85,15372 \\
UCs & 5 & 87,10229 \\
Fito + UCs (sem interação) & 8 & 212,18388 \\
Fito * UCs (com interação) & 10 & 155,63926 \\
\hline
\end{tabular}




\section{DISCUSSÃO}

A fauna das UCs menos preservadas do estudo, Parque Estadual do Araguaia e Refúgio de Vida Silvestre das Veredas do Oeste Baiano, apresentou significativamente mais espécies em relação à fauna das demais localidades amostradas. Esse resultado pode ser explicado pela hipótese do distúrbio intermediário, que prediz maior diversidade de espécies em ambientes cuja frequência (e provavelmente a intensidade) de distúrbios é intermediária, impedindo que a comunidade alcance um clímax que tende a ser menos diverso em espécies devido à exclusão competitiva (CoNNELl, 1978). Nas UCs classificadas como menos preservadas neste estudo, as fontes de distúrbios antrópicos observadas foram a criação extensiva de gado e a agricultura. No caso do Parque Estadual do Araguaia, o alagamento periódico e natural de partes de sua área durante a estação chuvosa também gera um mosaico de manchas em diferentes estágios de sucessão ecológica na paisagem, em que cada mancha provavelmente possui composição vegetal distinta. Isso, consequentemente, influencia a diversidade e composição das próprias mariposas, que usam as plantas como recurso (alimento e abrigo). A maior riqueza dessas mariposas em locais menos preservados corrobora outros trabalhos que constataram que Arctiinae, em particular a subtribo Phaegopterina, é mais diversa em ambientes em estágios iniciais e intermediários de sucessão, comparado a florestas primárias (KITCHING et al., 2000; HILT \& FIEDLER, 2005, 2006). Inclusive este táxon é considerado um bom indicador de ambientes tropicais alterados (KITCHING et al., 2000; HiLt \& FiedLER, 2005).

As fitofisionomias com estrutura vegetal mais complexa, cerradão e mata semidecídua, apresentaram maior riqueza de espécies. Geralmente hábitats estruturalmente complexos apresentam maior riqueza de espécies (BAZZAZ, 1975; MoReno et al., 2014), como predito pela hipótese da complexidade ambiental (BAZZAZ, 1975), já que fornecem maior variedade de microhábitats, amplitude microclimática e mais oportunidades de refúgios contra os inimigos naturais (BAZZaZ, 1975; Price et al., 1980; SANDers et al., 2008), possibilitando que maior quantidade de espécies coexista e, consequentemente, que a diversidade alfa aumente.

A fauna de mariposas dos pontos da mata semidecídua foi dissimilar em relação a campo cerrado e cerrado sensu stricto. Isto se deve ao fato de que áreas de mata apresentam composição de espécies vegetais, microclima e tipos de solo distintos das fitofisionomias do cerrado sensu lato (Oliveira-Filho \& RATter, 2002). Contudo, as áreas de mata foram similares ao cerradão. Este resultado pode ser explicado pelo fato de que o cerradão é uma fitofisionomia de transição, que engloba tanto espécies vegetais do cerrado sensu lato, quanto espécies florestais (Oliveira-Filho \& Ratter, 2002), o que se reflete na composição de espécies destas mariposas.

De modo geral, a composição de espécies entre as Unidades de Conservação foi bem dissimilar, principalmente entre o Parque Estadual do Araguaia e Parque Nacional Grande Sertão Veredas. Apenas as faunas do Parque Nacional das Nascentes do Rio Parnaíba e da Estação Ecológica Serra Geral do Tocantins foram similares entre si. Acreditamos que esta alta semelhança em relação à fauna dessas duas últimas UCs pode ser explicada pela similaridade do tipo de vegetação e também pela proximidade geográfica entre elas.

Até onde sabemos, a fauna de Arctiinae nunca havia sido amostrada nestas cinco UCs. Este trabalho contribuiu para o aumento dos registros de distribuição de 83 espécies, com 64 dessas exclusivas de alguma das UCs, o que reforça a importância da intensificação dos estudos nestas UCs, e em seus entornos. Também seria interessante investigar com maior profundidade quais os fatores que podem influenciar a maior riqueza de Arctiinae nas localidades menos preservadas, assim como realizar inventários mais prolongados.

Agradecimentos. A Laura Braga, Filipe Viegas, Vinicius Neves e Pedro da Silva pelo auxílio nas amostragens. Ao Diogo B. Provete pela revisão do resumo em inglês. Aos diretores e funcionários das UCs por todo apoio logístico. Carolina Moreno, Flávio Magalhães e Luiz Rezende receberam bolsa do CNPq. Esse projeto integra a RedeLep (CNPq, processo 563332/2010-7).

\section{REFERÊNCIAS BIBLIOGRÁFICAS}

Bazzaz, F. A. 1975. Plant species diversity in old-field successional ecosystems in Southern Illinois. Ecology 56:485-488.

Bolker, B. 2007. Ecological Models and data in R. Princeton, Princeton University Press, 508p.

Cavalcanti, R. B. \& Joly, C. A. 2002. Biodiversity and conservation priorities in the Cerrado region. In: OliveIRA, P. S. \& MARQuis, R. J. eds. The Cerrados of Brazil. Ecology and natural history of a Neotropical savanna. New York, Columbia University Press, p. 351-367.

Connell, J. H. 1978. Diversity in tropical rain forests and coral reefs. Science 199:1302-1310.

Ferro, V. G. \& Diniz, I. R. 2010. Riqueza e composição de mariposas Arctiidae (Lepidoptera) no Cerrado. In: DinIz, I. R.; MarinhoFilho, J.; Machado, R. B. \& Cavalcanti, R. B. eds. Cerrado, Conhecimento científico quantitativo como subsídio para ações de conservação. Brasília, Thesaurus, p. 255-313.

Ferro, V. G.; Melo, A. S. \& Diniz, I. R. 2010. Richness of tiger moths (Lepidoptera: Arctiidae) in the Brazilian Cerrado: how much do we know? Zoologia 27:725-731.

HAMmER, Ø.; HARPER, D. A. T. \& RYAN, P. D. 2001. PAST: Paleontological Statistics software package for education and data analysis. Palaeontologia Electronica 4:1-9.

Hampson, G. F. 1898. Catalogue of the Lepidoptera Phalaenae in the British Museum. London, Order of the Trustees. 559p.

1900. Catalogue of the Lepidoptera Phalaenae in the British Museum. London, Order of the Trustees. 589p.

. 1901. Catalogue of the Lepidoptera Phalaenae in the British Museum. London, Order of the Trustees. 690p.

. 1914. Catalogue of the Lepidoptera Phalaenae in the British Museum. London, Order of the Trustees. 858p.

HePpNER, J. B. 1991. Faunal regions and the diversity of Lepidoptera. Tropical Lepidoptera 2:1-85.

Hilt, N. \& FiedLER, K. 2005. Diversity and composition of Arctiidae moth ensembles along a successional gradient in the Ecuadorian Andes. Diversity and Distributions 11:387-398.

.2006. Arctiidae moth ensembles along a successional gradient in the Ecuadorian montane rain forest zone: how different are subfamilies and tribes? Journal of Biogeography 33:108-120. 
Kitching, R. L.; Orr, A. G.; Thalib, L.; Mitchell, H.; Hopkins, M. S. \& Graham, A. W. 2000. Moth assemblages as indicators of environmental quality in remnants of upland Australian rain forest. Journal of Applied Ecology 37:284-297.

Moreno, C.; Cianciaruso, M. V.; Sgarbi, L. G. \& Ferro, V. G. 2014. Richness and composition of tiger moths (Erebidae: Arctiinae) in a Neotropical savanna: are heterogeneous habitats richer in species? Natureza \& Conservação 12:138-143.

Oliveira-Filho, A. T. \& RatTER, J. A. 2002. Vegetation physiognomies and Woody flora of the Cerrado biome. In: OLIVEIRA, P. S. \& MARQUis, R. J. The Cerrados of Brazil. Columbia University Press, New York. $367 \mathrm{p}$.

Piñas-Rubio, F. \& Manzano, P. I. 2003. Mariposas del Ecuador, Arctiidae, Subfamília: Ctenuchinae. Quito, Conpañia de Jesús. 95p.

Piñas-Rubio, F.; Rab-Green, S.; Onore, G. \& Manzano, P. I. 2000. Mariposas Del Ecuador. Butterflies \& moths of Ecuador. Family: Arctiidae, Subfamilias: Arctiinae y Pericopinae. Quito, Pontificia Universidad Católica del Ecuador. 25p.
Price, P. W.; Bouton, C. E.; Gross, P.; McPheron, B. A.; Thompson, J. N. \& NeIs, A. E. 1980. Interactions among three trophic levels: influence of plants on interactions between insect herbivores and natural enemies. Annual Review of Ecology, Evolution, and Systematics 11:41-65.

Sanders, D.; Nickel, H.; Grutzner, T. \& Platner, C. 2008. Habitat structure mediates top-down effects of spiders and ants on herbivores. Basic and Applied Ecology 9:152-160.

Silveira-Neto, S. \& Silveira, A. C. 1969. Armadilha luminosa modelo "Luiz de Queiroz". O Solo 61:19-21.

The R Development Core Team. 2010. R: a language and environment for statistical computing. Vienna, R Foundation for Statistical Computing. Disponível em: $<$ http://www.R-project.org $>$. Acessado em: 20.07.2014.

Watson, A. \& Goodger, D. T. 1986. Catalogue of the Neotropical tigermoths. Occasional Papers on Systematic Entomology 1:1-70. 